\title{
Diverse laboratory colonies of Aedes aegypti harbor the same adult midgut bacterial microbiome
}

\author{
Laura B. Dickson ${ }^{1 *}$, Amine Ghozlane ${ }^{2,3}$, Stevenn Volant ${ }^{2}$, Christiane Bouchier ${ }^{3}$, Laurence Ma ${ }^{3}$, Anubis Vega-Rúa ${ }^{4}$, \\ Isabelle Dusfour ${ }^{5}$, Davy Jiolle ${ }^{6,7}$, Christophe Paupy ${ }^{6,7}$, Martin N. Mayanja $^{8}$, Alain Kohl ${ }^{9}$, Julius J. Lutwama ${ }^{8}$, \\ Veasna Duong ${ }^{10}$ and Louis Lambrechts ${ }^{1 *}$
}

\begin{abstract}
Background: Host-associated microbes, collectively known as the microbiota, play an important role in the biology of multicellular organisms. In mosquito vectors of human pathogens, the gut bacterial microbiota influences vectorial capacity and has become the subject of intense study. In laboratory studies of vector biology, genetic effects are often inferred from differences between geographically and genetically diverse colonies of mosquitoes that are reared in the same insectary. It is unclear, however, to what extent genetic effects can be confounded by uncontrolled differences in the microbiota composition among mosquito colonies. To address this question, we used 165 metagenomics to compare the midgut bacterial microbiome of six laboratory colonies of Aedes aegypti recently derived from wild populations representing the geographical range and genetic diversity of the species.

Results: We found that the diversity, abundance, and community structure of the midgut bacterial microbiome was remarkably similar among the six different colonies of Ae. aegypti, regardless of their geographical origin. We also confirmed the relatively low complexity of bacterial communities inhabiting the mosquito midgut.

Conclusions: Our finding that geographically diverse colonies of Ae. aegypti reared in the same insectary harbor a similar gut bacterial microbiome supports the conclusion that the gut microbiota of adult mosquitoes is environmentally determined regardless of the host genotype. Thus, uncontrolled differences in microbiota composition are unlikely to represent a significant confounding factor in genetic studies of vector biology.
\end{abstract}

Keywords: Mosquito, Microbiota, Vectorial capacity, Metagenomics

\section{Background}

The mosquito, Aedes aegypti, is the main vector of several medically important arboviruses such as Zika, dengue, chikungunya, and yellow fever viruses worldwide. Dengue viruses alone are responsible for 390 million human infections each year [1]. In the absence of vaccines or specific therapeutics for most arboviruses, controlling mosquito vector populations is the primary disease prevention strategy [2]. With the rise of insecticide resistance, the development of novel entomological interventions is underway [3, 4]. Critical to

\footnotetext{
* Correspondence: laura.dickson@pasteur.fr; louis.lambrechts@pasteur.fr ${ }^{1}$ Insect-Virus Interactions Group, Department of Genomes and Genetics, Institut Pasteur, CNRS UMR 2000, Paris, France

Full list of author information is available at the end of the article
}

the development of these new vector control methods is an improved understanding of the biology of mosquito vectors such as Ae. aegypti [5].

Over the last few decades, research efforts have focused on trying to elucidate the genetic [6-8] and environmental [9-12] factors that contribute to variation in the ability of Ae. aegypti to transmit human pathogens. Only in recent years, however, has the importance of the microbiota (i.e. host-associated microbes) emerged in vector biology. The gut bacterial microbiota in particular, influences multiple aspects of the mosquito's biology including vector competence $[13,14]$ and has become a topic of extensive research. Manipulation of the bacterial species present in the mosquito midgut has been shown to either increase or decrease the amounts of dengue 
virus, chikungunya virus, or Plasmodium falciparum [1519]. The composition of the microbiome (i.e. the collective genomes of the microbiota) found in the midgut of mosquitoes is highly variable and dependent on the environment [20-23] and life stage [24-26].

To identify mosquito genetic components of vectorial capacity, researchers often use genetically diverse colonies of mosquitoes reared in the same environment. Observed differences in the vectorial capacity of genetically diverse laboratory colonies is generally attributed to host genetics, and not to potential differences in the gut microbiota, but it remains unclear whether colonyspecific differences in microbiome could confound genetic studies. It was recently shown that the gut microbiota can be disrupted by genetic modification of mosquitoes [27]. In more natural insect systems such as the relationship between aphids, intracellular bacteria, and parasitic wasps, bacterial symbionts and not the aphid genotype drive the specificity of the interactions between the aphid and the parasitic wasp [28-30]. In other insects, it has been demonstrated that the gut microbiota contributes to host genotype by parasite genotype interactions [31], suggesting that differences in the microbiota should be considered as an additional factor when elucidating the host genetic contribution to a specific trait.

In Ae. aegypti, previous observations of bacterial taxa specific to certain mosquito lines reared in the same insectary $[32,33]$ raise the question whether differences in gut microbiota could confound interpretation of phenotypic differences among mosquito colonies. To address this question, we used a targeted metagenomics approach to compare the gut microbiome between six recent colonies of Ae. aegypti derived from wild populations representing the geographical range and genetic diversity of the species.
We performed a comprehensive metagenomics analysis including comparison of bacterial diversity within and between samples as well as identifying bacterial genera that are differentially abundant between colonies. Our results provide empirical evidence that adult Ae. aegypti mosquitoes reared in the same insectary harbor a similar gut bacterial microbiome, regardless of their geographic origin.

\section{Methods}

\section{Mosquito colonies and sample preparation}

Six Ae. aegypti colonies were chosen to maximize their genetic differences based on the geographical distribution of their populations of origin (Fig. 1, Table 1). Eggs from each of these colonies were simultaneously hatched in dechlorinated tap water under reduced air pressure for $1 \mathrm{~h}$ and 200 first-instar larvae from each colony were sorted into $24 \times 34 \times 9 \mathrm{~cm}$ plastic trays. The larvae were fed on a standard diet of Tetramin fish food (Tetra) every other day until pupation. Immediately following emergence, adults (males and females) were randomly separated into three replicate cages per mosquito colony. They were maintained under standard insectary conditions $\left(28^{\circ}\right.$ $\mathrm{C}, 70 \%$ relative humidity and $12 \mathrm{~h}$ light: $12 \mathrm{~h}$ dark cycle) for 4-6 days and allowed to mate and feed on sugar.

Midguts were dissected from adult females under sterile conditions in a biosafety cabinet. Each mosquito was surface sterilized in $70 \%$ ethanol for 3-5 min and washed three times in sterile $1 \times$ phosphate-buffered saline (PBS). Midguts were dissected in a drop of sterile $1 \times$ PBS and DNA from individual midguts was extracted as previously reported [9]. Briefly, individual midguts were ground in $300 \mu \mathrm{l}$ of $20 \mathrm{mg} / \mathrm{ml}$ lysozyme dissolved in Qiagen ATL buffer (Hilden, Germany) in a sterile tube containing grinding beads. The samples were homogenized for two

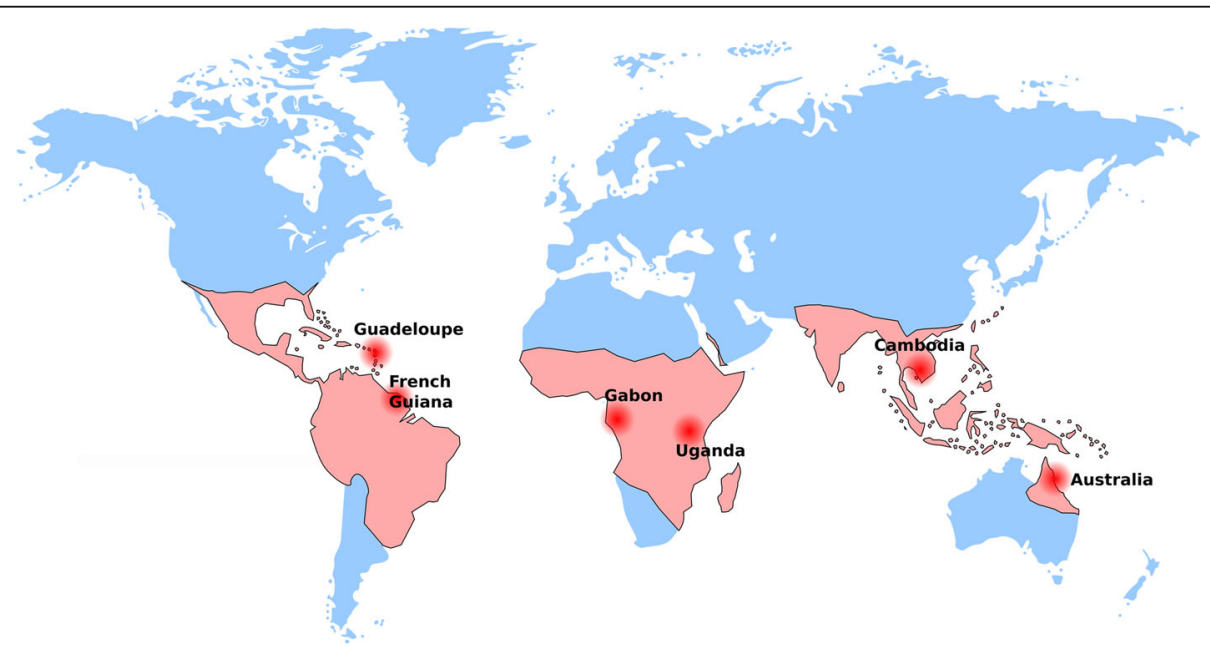

Fig. 1 World map showing the origin of the Ae. aegypti colonies used in the study overlaid with the approximate global distribution of Ae. aegypti adapted from Kraemer et al. [48, 49]. The colonies were initiated on different years and represent different generation times in the laboratory (Table 1) 
Table 1 Aedes aegypti colonies included in this study. The country and place of origin, year of collection, and number of generations spent in the laboratory prior to the study are shown

\begin{tabular}{llll}
\hline Country & Locality & Year & Generation \\
\hline Australia & Cairns & 2013 & 10 \\
Cambodia & Phnom Penh & 2015 & 7 \\
French Guiana & Cayenne & 2015 & 4 \\
Gabon & Bakoumba & 2014 & 10 \\
Guadeloupe & Saint François & 2015 & 5 \\
Uganda & Zika & 2016 & 3 \\
\hline
\end{tabular}

rounds of $30 \mathrm{~s}$ at 6,700 rpm (Precellys 24, Bertin Technologies, Montigny-le-Bretonneux, France) and DNA was extracted following the Qiagen DNeasy recommended pre-treatment protocol for Gram-positive bacterial samples. To control for contamination of bacteria introduced during the midgut dissections, DNA extractions, and PCR steps, negative controls were made by extracting DNA from blank $1 \times$ PBS that was used during the washing steps and by performing negative PCR reactions. DNA extractions were performed in two batches on different days.

\section{5 sequencing}

Custom-made PCR primers were designed to amplify the hypervariable V5-V6 region of the bacterial $16 \mathrm{~S}$ ribosomal RNA gene from midguts as previously described [9]. Purified DNA from each midgut sample was amplified in triplicate by 40 cycles of PCR using Expand High-Fidelity polymerase (Sigma-Aldrich, St. Louis, MI, USA) following manufacturer instructions. To improve PCR sensitivity, $0.15 \mu \mathrm{l}$ T4gene 32 and $0.5 \mu \mathrm{l} 20 \mathrm{mg} / \mathrm{ml}$ bovine serum albumin (BSA) were added per reaction with $6 \mu \mathrm{l}$ of template DNA. The three PCR reactions were pooled and the PCR products purified using Agencourt AMPure XP magnetic beads (Beckman Coulter, Brea, CA, USA). The purified PCR products were quantified by Quant-iT PicoGreen dsDNA fluorometric quantification (Thermo Fisher Scientific, Waltham, MA, USA) and pooled for sequencing in paired-end on the Illumina MiSeq platform using the 500-cycle v2 chemistry (Illumina, San Diego, CA, USA). On average, 16-18 individual midguts (5-6 individuals per adult replicate cage) were sequenced per $A$. aegypti colony. In order to achieve enough reads per sample, the sequencing was done in two separate runs. Libraries from each colony and each replicate were dispersed evenly between the two sequencing runs. Five libraries were removed from further analysis due to a low number of reads. Raw sequences were deposited to the European Nucleotide Archive under accession number PRJEB22905.

\section{Data analysis}

To account for possible contamination at various steps in the sample-processing pipeline, the sequencing reads were corrected with the reads from the negative controls. The sequencing reads from each sample were mapped to the reads found in the negative controls using Bowtie v.2.2.9 [34]. Reads that mapped to reads in the negative controls were removed from the analysis. Read filtering, operational taxonomic unit (OTU) clustering and annotation were performed with the MASQUE pipeline (https://github.com/aghozlane/masque) as previously described [35]. A total of 2679 OTUs were obtained at $97 \%$ sequence identity threshold. Genus richness and Shannon diversity index were compared by analysis of variance (ANOVA). All other statistical analyses were performed with SHAMAN (shaman.c3bi.pasteur.fr) as previously described [9]. Briefly, the normalization of OTU counts was performed at the OTU level using the DESeq2 normalization method. After normalization, six individuals were removed due to low size factors. In SHAMAN, a generalized linear model (GLM) was fitted and vectors of contrasts were defined to determine the significance in abundance variation between sample types. The GLM included the main effect of the Ae. aegypti colony, the main effect of replicate cage, the main effect of sequencing run and the interaction between colony and replicate. The resulting $P$-values were adjusted for multiple testing according to the Benjamini and Hochberg procedure [36]. Principal coordinates analysis ( $\mathrm{PCoA})$ was performed with the ade4 R package (v.1.7.6) using a Bray-Curtis dissimilarity matrix. Permutational multivariate analysis of variance (PERMANOVA) was performed in the vegan $\mathrm{R}$ package (v.2.4.3) as a distance-based method to test the statistical significance of the association between bacterial community structure and mosquito colony.

To confirm the OTU-based results with an OTUindependent method, a dissimilarity matrix was generated with the SIMKA software [37]. Reads with a positive match against the sequences assembled from the negative controls were removed using Bowtie v.2.2.9 [34]. Then, $k$-mers of size 32 and occurring at least greater than two times were identified with SIMKA. Bray-Curtis dissimilarity was estimated for $k$-mer abundance between each sample.

\section{Results}

To test if laboratory colonies of natural populations of Ae. aegypti differ in the diversity and composition of their gut microbiome, the V5-V6 variable region of the $16 \mathrm{~S}$ ribosomal RNA gene was sequenced in 16-18 individual adult female midguts from each of six recent colonies of Ae. aegypti. The six colonies originated from wild populations that represent the geographical range and genetic diversity of the species (Fig. 1) and had 
spent from three to ten generations in the laboratory prior to this study (Table 1). The experimental design included three replicate adult cages per colony and the individual libraries were randomized across two DNA extraction batches and two separate sequencing runs. Individual midguts were aseptically dissected from nulliparous, 4- to 6-day-old females that had been allowed to mate and feed on sugar following emergence. Out of the 96 individual gut microbiomes sequenced, 2679 operational taxonomic units (OTUs) representing 587 different bacterial genera were identified. Rarefaction curves showed that a sufficient number of sequencing reads was achieved to comprehensively characterize the bacterial communities in the midgut (Additional file 1: Figure S1).

To determine if the gut microbiome of each Ae. aegypti colony varies in the diversity of bacterial species present, the within-colony diversity was evaluated by determining the genus richness and the Shannon diversity index. No differences in the levels of richness (Fig. 2a) or in Shannon diversity index (Fig. 2b) were observed between the colonies (ANOVA: $F_{(5,90)}=1.125, P=$ 0.353 and $F_{(5,90)}=0.522, P=0.759$, respectively). In addition, the taxonomical abundance of bacteria was highly similar between the colonies, indicating that the dominant bacterial genera in the midgut are not dependent on the colony (Fig. 3).

To identify dissimilarities in the bacterial community structure between the gut microbiome of laboratory colonies of Ae. aegypti, principal coordinates analysis (PCoA) was performed based on a Bray-Curtis dissimilarity matrix. The PCoA showed that the bacterial community structures of all six colonies were highly similar to each other $(P=0.752)$ (Fig. 4a). In addition, no differences in the bacterial community structure were observed between the replicate cages of each colony, however the bacterial community structure differed between sequencing runs (Additional file 2: Figure S2). The reason for the run effect is unclear but it could reflect preferential clustering of specific sequences on the flow cell. The similar structure of bacterial communities among colonies was confirmed with an alternate analysis that does not rely on OTU counts, but instead groups sequences based on $k$-mers. In agreement with the OTU-based analysis, this analysis showed that the bacterial communities do not cluster based on the colony of origin, but instead based on technical factors such as the sequencing run and the DNA extraction batch (Additional file 3: Figure S3). Although the community structure of the gut microbiome was similar across colonies overall, we tested whether some specific bacterial taxa were differentially abundant. Out of the 587 bacterial genera identified, only zero to six genera were differentially abundant in pairwise comparisons of the six colonies (Additional file 4: Table S1, Fig. 4b) resulting in $98-100 \%$ similarity in the abundance of genera present between colonies.

\section{Discussion}

We performed a $16 S$ metagenomics analysis to compare the midgut microbiome of six recent colonies of Ae. aegypti reared in the same insectary environment. The six colonies were chosen to maximize their genetic differences based on the geographical distribution of their wild populations of origin. Although these colonies represent different genetic backgrounds and different generation times in the laboratory (Table 1), the gut microbiome was highly similar among all six colonies. We did not observe any differences in the diversity of the bacterial communities or in the bacterial community structure within the gut. The taxonomical abundance was also similar between the colonies with $98-100 \%$ identity in the abundance of bacterial genera present
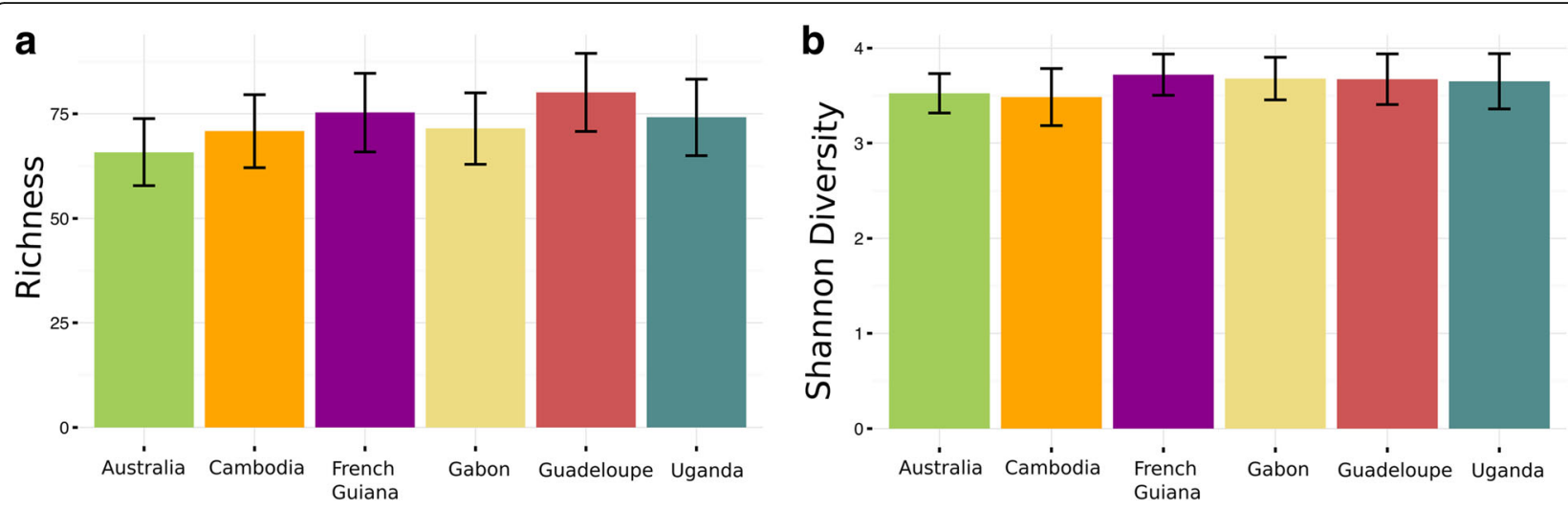

Fig. 2 Genetic diversity of the gut bacterial communities is similar between diverse colonies of Ae. aegypti. The genus richness (a) and Shannon diversity index (b) were calculated for each colony representing 16-18 individual midguts from 3 replicate cages dissected 4-6 days after adult emergence. Genus richness is the number of bacterial genera identified in each colony. The Shannon diversity index accounts for the relative abundance of each bacterial genus. Error bars represent $95 \%$ confidence intervals. No difference in richness (ANOVA: $\left.F_{(5,90)}=1.125, P=0.353\right)$ or in Shannon index (ANOVA: $F_{(5,90)}=0.522, P=0.759$ ) was detected between colonies 


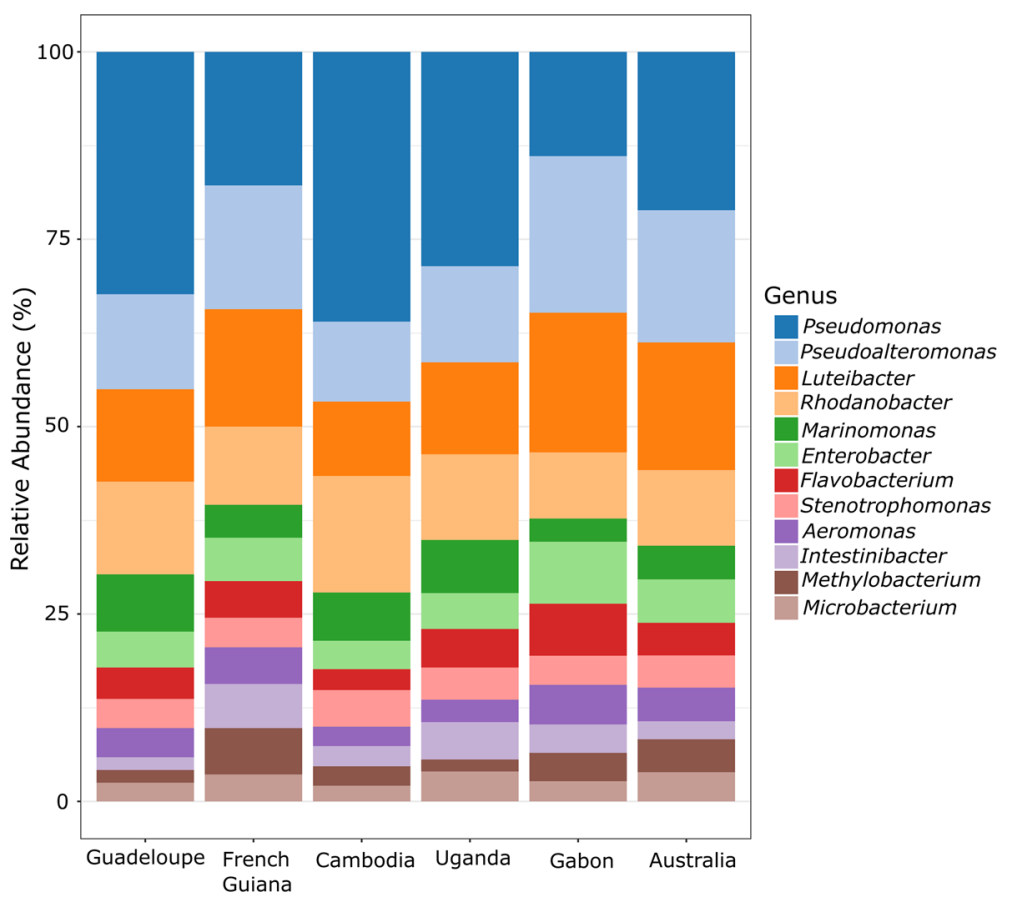

Fig. 3 The dominant bacterial genera found in the midgut are similar among diverse colonies of Ae. aegypti. The abundance of the 12 most abundant genera is shown for each colony representing 16-18 individual midguts from 3 replicate cages dissected 4-6 days after adult emergence. Bacterial genera were assigned to OTUs clustered with a 97\% cut-off using the SILVA database (https://www.arb-silva.de)

between colonies. The data also confirmed the relatively low complexity of bacterial communities typically found in the gut of insects $[38,39]$.

Other studies that have compared the midgut microbiome of various laboratory colonies of Ae. aegypti observed differences in the taxonomical identification of specific bacterial species [32, 33]. Although these studies reported differences in the abundance of specific taxa between colonies of Ae. aegypti, no difference in the bacterial community structure was in fact observed. Furthermore, the colonies tested in previous studies have been maintained in the laboratory for five to 80 years before their microbiome was examined. It is possible that large differences in the number of generations spent in the laboratory between these studies and ours, resulted in our different observations. Possibly, preferential
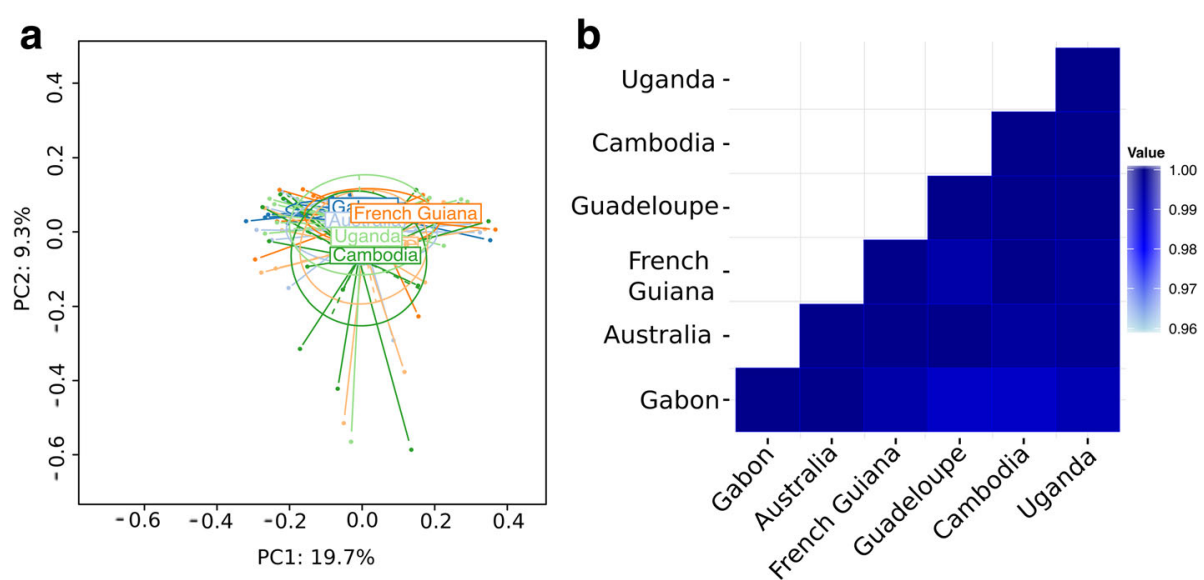

Fig. 4 The midgut bacterial community structure is similar between diverse colonies of Ae. aegypti. Bacterial community structures between colonies are compared by (a) principal coordinates analysis (PCOA) and (b) pairwise differential abundance analysis. PCoA is based on a Bray-Curtis dissimilarity matrix and indicates a lack of overall differences (PERMANOVA: $P$-value $=0.752$ ). Results of differential abundance analysis are shown for each pair of colonies as the proportion of all bacterial genera identified $(n=587)$ that were non-significantly differentially abundant (Wald test) after correction for multiple testing 
associations between mosquito genotypes and specific laboratory bacteria may evolve over a long colonization history. This hypothesis remains to be tested.

Researchers often use genetically diverse colonies of mosquitoes reared in the same environment to identify mosquito genetic components of vectorial capacity. In such studies, differences in microbiota could confound interpretation of phenotypic differences among mosquito colonies. The present study does not support this hypothesis in the case of Ae. aegypti. While this may be the case in some insect systems [28-31], our study provides evidence that the midgut microbiome of colonized Ae. aegypti is highly similar and most likely will not confound genetic studies of vector biology.

It is well accepted that Ae. aegypti from sub-Saharan Africa belong to a different phylogenetic cluster than pan-tropical Ae. aegypti from elsewhere in the world [40-44]. At a more local scale, populations of Ae. aegypti sampled from distinct locations are usually genetically distinct [45-47]. Accordingly, we assumed that the colonies that we tested in fact represent various genotypes of Ae. aegypti. However, we could not directly ascertain the correlation between mosquito genotype and microbiome because we did not genotype the Ae. aegypti colonies used in this study. It is likely that founder effects and adaptation to laboratory conditions modified the genetic composition of the colonies relative to their wild populations of origin. Nevertheless, our results show that laboratory-bred Ae. aegypti share the same bacterial microbiome regardless of the genetic differences of their populations of origin. Although this finding suggests that the environment plays a dominant role in shaping the gut microbiome of Ae. aegypti, this may not be the case in a more natural system. One can imagine that within a given environment, the mosquito genotype may influence the composition of the midgut microbiome and this should be explored further.

Although the vast majority (>98\%) of bacterial genera were present at statistically similar abundance between colonies (Fig. 4b), there were a few genera that were differentially abundant in pairwise comparisons (Additional file 4: Table S1). For example, the Gabon colony had a lower abundance of Arenimonas and Pseudonocardia than three other colonies, and a lower abundance of Aeribacillus than four other colonies. Likewise, the Australia colony had a lower abundance of Ilumatobacter than three other colonies. While none of these genera have been associated with vectorial capacity in mosquitoes [13], whether these differences are biologically meaningful remains to be determined. We did not detect an association between the number of generations spent in the laboratory and specific bacterial genera that were differentially abundant between colonies. In fact, no bacterial genus was differentially abundant across pairs of colony that did not share a member (Additional file 4: Table S1).

A limitation of our study was that we only dissected midguts at one time point. Recent results from Short et al. [33] suggest that differences between colonies may exist at different times following adult emergence. It is possible that differences in the gut microbiome between our colonies would have been observed if we had sampled the midguts sooner or later after adult emergence. Since our primary goal was to determine how the gut microbiome of our colonies may impact studies of vector competence, we chose a time point after adult emergence that related to the time when an infectious blood meal is usually offered in vector competence assays.

One potentially important implication of our results is that the same mosquito strain reared in different laboratories might display different phenotypes due to a different gut microbiota. We found that the gut microbiome of mosquito colonies was entirely determined by the insectary environment regardless of the mosquito genotype. It follows that the same mosquito strain exposed to a different environment could host a different gut microbiota. This could undermine the relevance of reference strains that are shared by different laboratories. It will be interesting in future studies to compare the gut bacterial microbiome of the same mosquito strain reared in different insectaries.

\section{Conclusions}

Our finding that geographically diverse colonies of $A e$. aegypti reared in the same insectary harbor a similar gut bacterial microbiome supports the conclusion that the gut microbiota of adult mosquitoes is environmentally determined, regardless of the host genotype. Thus, uncontrolled differences in microbiota composition are unlikely to represent a significant confounding factor in genetic studies of vector biology.

\section{Additional files}

Additional file 1: Figure S1. Rarefaction curves for the individual samples used in the analysis at the genus level. The curves show the number of detected bacterial genera as a function of the number of reads analyzed per sequencing library. Each curve represents a single midgut sample. (PNG $58 \mathrm{~kb}$ )

Additional file 2: Figure S2. The midgut bacterial communities are highly structured by sequencing run. The cluster dendrogram of individual midgut samples based on a Bray-Curtis dissimilarity matrix shows that sequencing run, and not the identity of the mosquito colony, determines bacterial community relatedness. Midgut samples are represented by numbers color coded by sequencing run. Dark blue samples were sequenced in the first run, whereas light blue samples were sequenced in the second run. (PNG $2300 \mathrm{~kb}$ )

Additional file 3: Figure S3. OTU-independent analysis of midgut bacterial community structure confirms the lack of clustering by colony. The heat map of Bray-Curtis dissimilarity index based on k-mer abundance and hierarchical clustering shows that the sequences are structured based on the sequencing run and the day of DNA extraction, not the 
laboratory colony of origin. Sample names are color coded by colony and labeled according to the DNA extraction batch ( $a$ and $b$ ) and the sequencing run $\left({ }^{*}\right.$ and +$)$. In the heat map, red color indicates high similarity, whereas green color indicates low similarity. (PNG $476 \mathrm{~kb}$ )

Additional file 4: Table S1. Identification of bacterial genera that are differentially abundant in pairwise comparisons of colonies. $P$-values were obtained with a Wald test are corrected for multiple testing. The lack of a comparison between two colonies indicates that no bacterial genera were significantly different between them. (XLSX $15 \mathrm{~kb}$ )

\section{Acknowledgements}

We are grateful to Gordana Rašić and Ary Hoffmann for providing the mosquito colony from Cairns and to Borin Peng for the mosquito collection in Phnom Penh. We thank Claire Valiente Moro, Guillaume Minard and the Lambrechts lab members for their insights.

\section{Funding}

This work was supported by Agence Nationale de la Recherche (grants ANR16-CE35-0004-01 and ANR-17-ERC2-0016-01 to LL), the French Government's Investissement d'Avenir program Laboratoire d'Excellence Integrative Biology of Emerging Infectious Diseases (grant ANR-10-LABX-62-IBEID to LL), the City of Paris Emergence(s) program in Biomedical Research (to LL), the European Union's Horizon 2020 research and innovation programme under ZikaPLAN grant agreement No 734584 (to LL), the European Union and Guadeloupe Region (Programme Opérationnel FEDER-Guadeloupe-Conseil Régional 20142020, grant 2015-FED-192 to AVR) and the United Kingdom Medical Research Council (grant MC_UU_12014 to AK). The Genomics Facility is a member of the "France Génomique" consortium (grant ANR10-INBS-09-08 to CB). The funders had no role in study design, data collection and interpretation, or the decision to submit the work for publication.

\section{Availability of data and materials}

The dataset generated during the current study is available in the European Nucleotide Archive under accession number PRJEB22905.

\section{Authors' contribution}

LBD and LL designed the study. AVR, ID, DJ, CP, MNM, AK, JJL and VD provided mosquito specimens to initiate colonies. $L B D$ carried out the experiments. $L B D, C B$ and $L M$ performed the sequencing. $L B D, A G$, SV and $L L$ analyzed the data. LBD and LL wrote the manuscript. All authors read and approved the final manuscript.

\section{Ethics approval and consent to participate}

Not applicable.

\section{Consent for publication}

Not applicable.

\section{Competing interests}

The authors declare that they have no competing interests.

\section{Publisher's Note}

Springer Nature remains neutral with regard to jurisdictional claims in published maps and institutional affiliations.

\section{Author details}

Insect-Virus Interactions Group, Department of Genomes and Genetics, Institut Pasteur, CNRS UMR 2000, Paris, France. ²Bioinformatics and Biostatistics Hub, C3BI, USR 3756 CNRS, Institut Pasteur, Paris, France. ${ }^{3}$ Genomics Facility - Biomics Pole, CITECH, Institut Pasteur, Paris, France. ${ }^{4}$ Laboratory of Medical Entomology, Environment and Health Unit, Institut Pasteur de la Guadeloupe, Guadeloupe, France. ${ }^{5}$ Vector Control and Adaptation, Institut Pasteur de la Guyane, Vectopole Amazonien Emile Abonnenc, Cayenne, French Guiana. ${ }^{6}$ MIVEGEC, IRD, CNRS, University of Montpellier, Montpellier, France. ${ }^{7}$ Centre International de Recherches Médicales de Franceville, Franceville, Gabon. ${ }^{8}$ Department of Arbovirology, Uganda Virus Research Institute, Entebbe, Uganda. ${ }^{9}$ MRC-University of Glasgow Centre for Virus Research, Glasgow, UK. ${ }^{10}$ Virology Unit, Institut Pasteur in Cambodia, Phnom Penh, Cambodia.
Received: 11 October 2017 Accepted: 6 March 2018

Published online: 27 March 2018

\section{References}

1. Bhatt S, Gething PW, Brady OJ, Messina JP, Farlow AW, Moyes $C L$, et al. The global distribution and burden of dengue. Nature. 2013;496(7446):504-7.

2. Wilder-Smith A, Gubler DJ, Weaver SC, Monath TP, Heymann DL, Scott TW. Epidemic arboviral diseases: priorities for research and public health. Lancet Infect Dis. 2017;17(3):e101-e6.

3. McGraw EA, O'Neill SL. Beyond insecticides: new thinking on an ancient problem. Nat Rev Microbiol. 2013;11(3):181-93.

4. Lambrechts L, Ferguson NM, Harris E, Holmes EC, McGraw EA, O'Neill SL, et al. Assessing the epidemiological effect of Wolbachia for dengue control. Lancet Infect Dis. 2015;15(7):862-6.

5. Lambrechts L, Knox TB, Wong J, Liebman KA, Albright RG, Stoddard ST. Shifting priorities in vector biology to improve control of vector-borne disease. Trop Med Int Health. 2009;14(12):1505-14.

6. Bosio CF, Fulton RE, Salasek ML, Beaty BJ, Black WC. Quantitative trait loci that control vector competence for dengue-2 virus in the mosquito Aedes aegypti. Genetics. 2000;156(2):687-98.

7. Bennett KE, Flick D, Fleming KH, Jochim R, Beaty BJ, Black WC. Quantitative trait loci that control denque-2 virus dissemination in the mosquito Aedes aegypti. Genetics. 2005;170(1):185-94.

8. Fansiri T, Fontaine A, Diancourt L, Caro V, Thaisomboonsuk B, Richardson JH, et al. Genetic mapping of specific interactions between Aedes aegypti mosquitoes and dengue viruses. PLoS Genet. 2013:9(8):e1003621.

9. Dickson LB, Jiolle D, Minard G, Moltini-Conclois I, Volant S, Ghozlane A, et al. Carryover effects of larval exposure to different environmental bacteria drive adult trait variation in a mosquito vector. Sci Adv. 2017:3(8):e1700585.

10. Lambrechts L, Paaijmans KP, Fansiri T, Carrington LB, Kramer LD, Thomas $M B$, et al. Impact of daily temperature fluctuations on dengue virus transmission by Aedes aegypti. Proc Natl Acad Sci USA. 2011;108(18):7460-5.

11. Westbrook CJ, Reiskind MH, Pesko KN, Greene KE, Lounibos LP. Larval environmental temperature and the susceptibility of Aedes albopictus Skuse (Diptera: Culicidae) to chikungunya virus. Vector Borne Zoonotic Dis. 2010;10(3):241-7.

12. Alto $B W$, Lounibos $L P$, Higgs $S$, Juliano $S A$. Larval competition differentially affects arbovirus infection in Aedes mosquitoes. Ecology. 2005;86(12):3279-88.

13. Minard G, Mavingui $P$, Moro CV. Diversity and function of bacterial microbiota in the mosquito holobiont. Parasit Vectors. 2013;6:146.

14. Hegde S, Rasgon JL, Hughes GL. The microbiome modulates arbovirus transmission in mosquitoes. Curr Opin Virol. 2015;15:97-102.

15. Cirimotich CM, Ramirez JL, Dimopoulos G. Native microbiota shape insect vector competence for human pathogens. Cell Host Microbe. 2011;10(4):307-10.

16. Ramirez JL, Short SM, Bahia AC, Saraiva RG, Dong Y, Kang S, et al. Chromobacterium Csp_P reduces malaria and dengue infection in vector mosquitoes and has entomopathogenic and in vitro anti-pathogen activities. PLoS Pathog. 2014:10(10):e1004398.

17. Ramirez JL, Souza-Neto J, Torres Cosme R, Rovira J, Ortiz A, Pascale JM, et al. Reciprocal tripartite interactions between the Aedes aegypti midgut microbiota, innate immune system and dengue virus influences vector competence. PLoS Negl Trop Dis. 2012;6(3):e1561.

18. Apte-Deshpande A, Paingankar M, Gokhale MD, Deobagkar DN. Serratia odorifera a midqut inhabitant of Aedes aegypti mosquito enhances its susceptibility to dengue-2 virus. PLoS One. 2012;7(7):e40401.

19. Apte-Deshpande AD, Paingankar MS, Gokhale MD, Deobagkar DN. Serratia odorifera mediated enhancement in susceptibility of Aedes aegypti for chikungunya virus. Indian J Med Res. 2014;139(5):762-8.

20. Zouache K, Raharimalala FN, Raquin V, Tran-Van V, Raveloson LH, Ravelonandro P, et al. Bacterial diversity of field-caught mosquitoes, Aedes albopictus and Aedes aegypti, from different geographic regions of Madagascar. FEMS Microbiol Ecol. 2011;75(3):377-89.

21. Coon KL, Brown MR, Strand MR. Mosquitoes host communities of bacteria that are essential for development but vary greatly between local habitats. Mol Ecol. 2016;25(22):5806-26.

22. Osei-Poku J, Mbogo CM, Palmer WJ, Jiggins FM. Deep sequencing reveals extensive variation in the gut microbiota of wild mosquitoes from Kenya. Mol Ecol. 2012;21 (20):5138-50.

23. Buck M, Nilsson LK, Brunius C, Dabire RK, Hopkins R, Terenius O. Bacterial associations reveal spatial population dynamics in Anopheles gambiae mosquitoes. Sci Rep. 2016;6:22806. 
24. Gimonneau G, Tchioffo MT, Abate L, Boissiere A, Awono-Ambene PH, Nsango SE, et al. Composition of Anopheles coluzzii and Anopheles gambiae microbiota from larval to adult stages. Infect Genet Evol. 2014;28:715-24.

25. Coon KL, Vogel KJ, Brown MR, Strand MR. Mosquitoes rely on their gut microbiota for development. Mol Ecol. 2014;23(11):2727-39.

26. Wang Y, Gilbreath TM 3rd, Kukutla P, Yan G, Xu J. Dynamic gut microbiome across life history of the malaria mosquito Anopheles gambiae in Kenya. PLoS One. 2011;6(9):e24767.

27. Pike A, Dong Y, Dizaji NB, Gacita A, Mongodin EF, Dimopoulos G. Changes in the microbiota cause genetically modified Anopheles to spread in a population. Science. 2017;357(6358):1396-9.

28. Oliver KM, Moran NA, Hunter MS. Variation in resistance to parasitism in aphids is due to symbionts not host genotype. Proc Natl Acad Sci USA. 2005;102(36):12795-800.

29. Sandrock C, Gouskov A, Vorburger C. Ample genetic variation but no evidence for genotype specificity in an all-parthenogenetic host-parasitoid interaction. J Evol Biol. 2010;23(3):578-85.

30. Vorburger C, Sandrock C, Gouskov A, Castaneda LE, Ferrari J. Genotypic variation and the role of defensive endosymbionts in an allparthenogenetic host-parasitoid interaction. Evolution. 2009;63(6):1439-50.

31. Koch H, Schmid-Hempel P. Gut microbiota instead of host genotype drive the specificity in the interaction of a natural host-parasite system. Ecol Lett. 2012;15(10):1095-103.

32. Charan SS, Pawar KD, Severson DW, Patole MS, Shouche YS. Comparative analysis of midgut bacterial communities of Aedes aegypti mosquito strains varying in vector competence to dengue virus. Parasitol Res. 2013;112(7):2627-37.

33. Short SM, Mongodin EF, MacLeod HJ, Talyuli OAC, Dimopoulos G. Amino acid metabolic signaling influences Aedes aegypti midgut microbiome variability. PLoS Negl Trop Dis. 2017;11(7):e0005677.

34. Langmead B, Salzberg SL. Fast gapped-read alignment with Bowtie 2. Nat Methods. 2012;9(4):357-9.

35. Quereda JJ, Dussurget O, Nahori MA, Ghozlane A, Volant S, Dillies MA, et al. Bacteriocin from epidemic Listeria strains alters the host intestinal microbiota to favor infection. Proc Natl Acad Sci USA. 2016;113(20):5706-11.

36. Hochberg $Y$, Benjamini Y. Controlling the false discovery rate: a practical and powerful approach to multiple testing. J Roy Stat Soc B. 1995:57(1):289-300.

37. Benoit G, Peterlongo P, Mariadassou M, Drezen E, Schbath S, Lavenier D, et al. Multiple comparative metagenomics using multiset k-mer counting. PeerJ Comput Sci. 2016;2:e94.

38. Engel P, Moran NA. The gut microbiota of insects - diversity in structure and function. FEMS Microbiol Rev. 2013;37(5):699-735.

39. Dillon RJ, Dillon VM. The gut bacteria of insects: nonpathogenic interactions. Annu Rev Entomol. 2004:49:71-92.

40. Tabachnick WJ, Powell JR. A world-wide survey of genetic variation in the yellow fever mosquito, Aedes aegypti. Genet Res. 1979;34(3):215-29.

41. Dickson LB, Campbell CL, Juneja P, Jiggins FM, Sylla M, Black WC. Exon-enriched libraries reveal large genic differences between Aedes aegypti from Senegal, West Africa, and populations outside Africa. G3. 2017;7(2):571-82

42. Brown JE, Evans BR, Zheng W, Obas V, Barrera-Martinez L, Egizi A, et al. Human impacts have shaped historical and recent evolution in Aedes aegypti, the dengue and yellow fever mosquito. Evolution. 2014:68(2):514-25.

43. Brown JE, McBride CS, Johnson P, Ritchie S, Paupy C, Bossin H, et al. Worldwide patterns of genetic differentiation imply multiple 'domestications' of Aedes aegypti, a major vector of human diseases. Proc Biol Sci. 2011;278(1717):2446-54.

44. Crawford JE, Alves JM, Palmer WJ, Day JP, Sylla M, Ramasamy R, et al. Population genomics reveals that an anthropophilic population of Aedes aegypti mosquitoes in West Africa recently gave rise to American and Asian populations of this major disease vector. BMC Biol. 2017;15(1):16.

45. Gloria-Soria A, Ayala D, Bheecarry A, Calderon-Arguedas O, Chadee DD, Chiappero M, et al. Global genetic diversity of Aedes aegypti. Mol Ecol. 2016; 25(21):5377-95.

46. Bosio CF, Harrington LC, Jones JW, Sithiprasasna R, Norris DE, Scott TW. Genetic structure of Aedes aegypti populations in Thailand using mitochondrial DNA. Am J Trop Med Hyg. 2005;72(4):434-42.

47. Garcia-Franco F, Munoz Mde L, Lozano-Fuentes S, Fernandez-Salas I, GarciaRejon J, Beaty BJ, et al. Large genetic distances among Aedes aegypti populations along the South Pacific coast of Mexico. Am J Trop Med Hyg 2002;66(5):594-8.

48. Kraemer MU, Sinka ME, Duda KA, Mylne AQ, Shearer FM, Barker CM, et al. The global distribution of the arbovirus vectors Aedes aegypti and Ae. albopictus. Elife. 2015;4:e08347.

49. Kraemer MU, Sinka ME, Duda KA, Mylne A, Shearer FM, Brady OJ, et al. The global compendium of Aedes aegypti and Ae. albopictus occurrence. Sci Data. 2015;2:150035

\section{Submit your next manuscript to BioMed Central and we will help you at every step:}

- We accept pre-submission inquiries

- Our selector tool helps you to find the most relevant journal

- We provide round the clock customer support

- Convenient online submission

- Thorough peer review

- Inclusion in PubMed and all major indexing services

- Maximum visibility for your research

Submit your manuscript at www.biomedcentral.com/submit
Biomed Central 